\title{
Modeling and Testing Friction Flexible Dampers: Challenges and Peculiarities
}

\author{
A. Fantetti ${ }^{1}$ (D) C. Gastaldi ${ }^{2}$. T.M. Berruti ${ }^{2}$ \\ Received: 7 November 2017 / Accepted: 11 April 2018 / Published online: 8 May 2018 \\ (C) The Author(s) 2018
}

\begin{abstract}
This paper deals with the dynamic of blades with strip dampers. The purpose is 1) to present the results of the dynamic numerical calculation, 2) to demonstrate the need for the experimental data on the blade-strip contact to be used as input to the calculation, 3) to propose a new test rig design to obtain them and 4) to test the key components of the new test rig. The forced responses of two blades coupled by a strip damper are calculated at different excitation and centrifugal force values. The dependence of the numerical results on the contact parameter values is confirmed in this significant reference case. The design of a new test rig is then proposed: both the blade frequency response function and the contact hysteresis cycles at the blade-strip contact are measured. It is shown how contact parameters can then be derived from experimental data. The main novelty of the test rig here proposed is the strip loading system, which simulates the uniform pressure distribution provided by the centrifugal force in real operating conditions. This loading system is non-contact and uses compressed air. Classical loading systems which see dead weights directly connected to the strip are assessed and their expected inadequacy is confirmed. The compressed air system is tested by measuring the pressure produced between strip and blades: pressure is uniform across the contact patch, constant in time and its mean value corresponds to realistic pressure values actually experienced by strip dampers during service.
\end{abstract}

Keywords Strip dampers · Numerical modeling $\cdot$ Test rig $\cdot$ Contact parameters $\cdot$ Forced response

\section{Introduction}

Due to the high modal density of realistic bladed disks and to the broad frequency content of the aerodynamic excitation forces, attaining a blade design which is resonance-free in the frequency range of interest is unfeasible. Since turbine blades do not benefit significantly from material hysteresis and aerodynamic damping, the current best option is to add external sources of damping, e.g. in the form of dry friction devices [1, 2]. Dry friction can be incorporated into the blade design, in the form of shrouds, lacing wires or zigzag pins. Alternatively, external devices such as solid dampers (available in several designs, cylindrical, curved flat, wedge damper, etc), thinstrip dampers [3, 4] or ring dampers [5, 6] can be added to minimize the resonant blade response. A detailed analysis

\footnotetext{
$\triangle$ A. Fantetti

a.fantetti@imperial.ac.uk

Imperial College London, London, UK

2 Politecnico di Torino, Turin, Italy
}

of the different sources of damping in turbo engines was performed in [2]. It was shown that the values of $Q$ (amplitude factor, inverse of the loss factor $\eta$ ) may vary as follows: 3000 to 10,000 for material damping, 50 to 140 for root damping, 180 to 2500 for shroud damping, and 15 to 250 for platform damping (external dry friction dampers). Dampers are especially effective (low values of Q) if bending modes are considered (also explored in this paper), while $Q$ values tend to increase for higher (torsional) modes. For this reason, the focus of the present investigation is on lower (bending) modes.

External dry friction dampers are then extensively used in turbine designs because they are not only very effective, but also easy to manufacture, install and substitute, relatively inexpensive and can withstand high temperatures. Among external dry friction dampers, the strip (or seal) dampers are thin flexible metallic strips which are positioned under the blade platforms of turbine bladed disks and their primary function is to seal the cooling air. They are pushed against the blade platforms by the centrifugal force. When relative motion takes place between strip and blade platforms, the friction forces dissipate vibrational energy and consequently 
the system response is damped. In this way the strips act also as friction dampers which introduce both damping and a constraint affecting significantly the blade natural frequencies [7].

The design of the strips, not only for sealing but also for damping purpose, represents a topic of recent interest for the turbine designers, as very few studies can be found in literature $[3,4]$.

In the past 15 years, the so called solid underplatform dampers (UPDs) have been extensively investigated [814]. Most of the proposed calculation methodologies for bulk UPDs use simplifying assumptions, such as neglecting the damper inertia or flexibility [14-18]. However, these assumptions, perfectly adequate for solid UPDs, cannot be applied to model strip dampers, as their high flexibility needs to be taken into account in the modeling process $[4$, 7]. Therefore, in this paper the strip damper is modeled using Finite Elements (FE) [4]. Furthermore, the most advanced state-of-the-art calculation methods are here applied to predict the dynamics of two blades and a strip damper between them. In detail, the nonlinear forced response is calculated with high computational efficiency in the frequency domain using the Multi-Harmonic Balance Method (MHBM) [19, 20], a reduction method is adopted to decrease the size of the blades [21] and the nonlinear equations are solved by an iterative solver using the analytical computation of the Jacobian matrix [20, 22]. Lastly, a novel technique presented in [22] ensures that the MHBM (an approximate method) offers results with an accuracy above a user-defined threshold.

Even if the calculated responses vary with the centrifugal to excitation force ratio following the expected pattern, it is shown that the computational results in terms of natural frequency and vibration amplitude strongly depend on the contact parameters values (friction coefficient, normal and tangential contact stiffnesses). This dependence has not been addressed in [3, 4] where a unique assumption on the contact parameter values has been used throughout the papers. This practice has potentially dangerous consequences as will be shown in a dedicated section of this paper. The contact parameters should be determined experimentally. However no record of this investigation performed on very flexible dampers can be found in literature. Furthermore, the results of similar investigation performed on solid UPDs [12-14, 23-27] cannot be applied here as normal loads and contact conditions of solid and flexible dampers differ.

In the past ten years, the AERMEC lab at the Polytechnic of Turin has devoted time and effort in the direct experimental investigation of friction parameters on different type of friction contacts [28-34]. By using this experience on different test rigs, the design of a new test rig tailored on strip dampers is here proposed.
The test rig allows measuring, at the same time, the frequency response of a blade with a strip and the contact hysteresis cycles desired for the direct determination of contact parameters.

Purpose of this paper is to:

- briefly present the numerically-calculated nonlinear dynamic response of blades with strip dampers to highlight its strong dependence on the contact parameters and, consequently, the need for an accurate estimate of their values;

- disclose the design of a novel test rig where both the frequency response function of the blade and the contact hysteresis cycles at the strip-blade contact can be measured;

- prove the feasibility of the rig by testing its key components:

- the systems to measure the contact forces and displacement at the contact;

- the loading system to simulate the centrifugal force on the strip.

While some kind of previous experience is available for the other key elements [33, 34] and competences [13, $22,32]$, the loading system of a flexible structure is an entirely novel challenge. Centrifugal load on solid UPDs is typically simulated through a dead weight system with wires (i.e. point loading at the damper center of mass): the high UPD bulk stiffness ensures that the centrifugal load is transmitted to the contact interfaces without deforming the UPD itself. If, as in the present case, the damper is flexible, point loading at the damper center of mass is not a viable option as it would not guarantee a uniform contact pressure and would actually deform the damper. A multi-point loading "distributed" across the contact interfaces is equally unacceptable as contact pressures would be "localized" about the loading points. Furthermore, any external device touching the strip would modify the contact area and strip stiffness. The real challenge, addressed in the last section of this paper, is to devise a loading system capable of:

- guaranteeing a uniform pressure distribution across the contact surface;

- avoiding contact with the strip damper since, as will be shown below, traditional contact loading systems change the surface contact conditions.

\section{Dynamic Equilibrium Equations}

The ultimate goal of this research is to be able to predict the dynamic behavior of a full disk with strip dampers, as shown in Fig. 1(a). A useful starting point is the investigation of two blades with one strip damper between them. This 

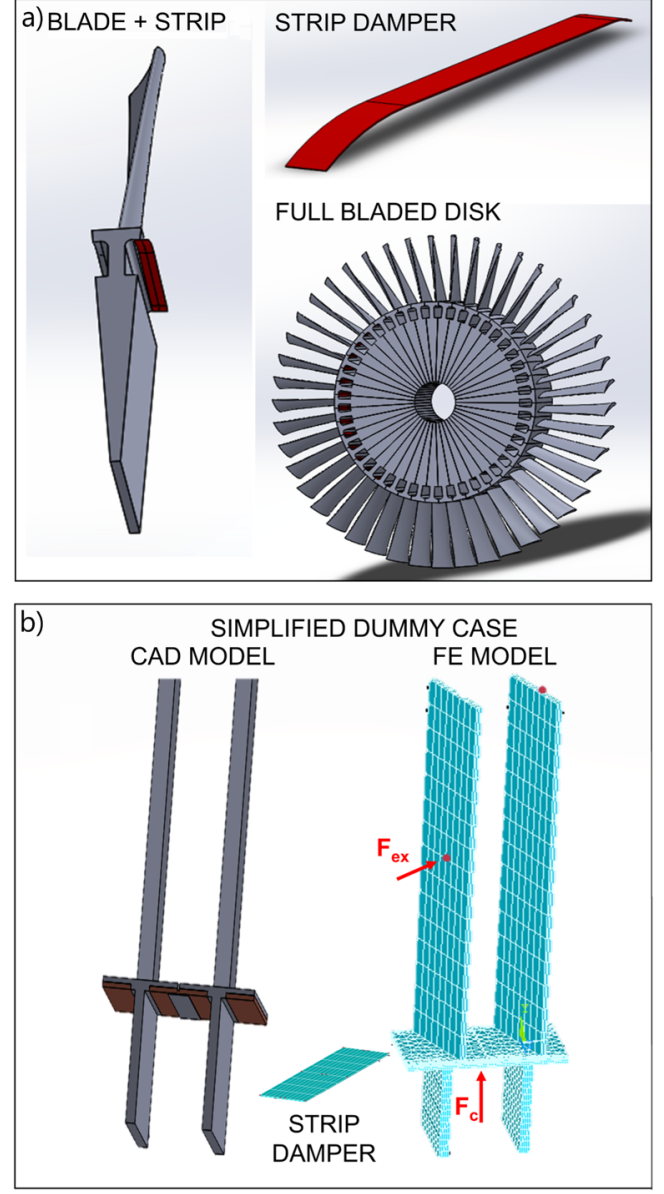

Fig. 1 (a) Blades with strip damper; (b) simplified case: dummy blades with a strip damper

simplified model is shown in Fig. 1(b) $)^{1}$. Blades and strip are separate structures that interact with each other only through the contact friction forces [4]. From a dynamic point of view, the blades with a strip damper can be considered as a unique system $[36,37]$ described by the following dynamic balance equations:

$M \ddot{\mathbf{x}}(t)+C \dot{\mathbf{x}}(t)+K \mathbf{x}(t)=\mathbf{F}_{\mathbf{e x}}(t)+\mathbf{F}_{\mathbf{c}}-\mathbf{F}_{\mathbf{n l}}(\mathbf{x}, \dot{\mathbf{x}}, t)$

Where:

- $\quad M, C$, and $K$ are the mass, damping and stiffness matrices of the system respectively. They are obtained by combining the mass, damping and stiffness matrices of both strip and blades. These matrices are extracted from the FE models of strip and blades after a Craig Bampton-Component Mode Synthesis (CB-CMS) reduction [21].

- $\quad \mathbf{x}$ is the vector of the DOFs of strip and blades. Due to the CB-CMS reduction, this vector includes both modal

\footnotetext{
${ }^{1}$ The simulation of the full bladed disk is easily obtained by imposing the well-known cyclic symmetric boundary conditions [35].
}

DOFs and the physical DOFs which are the displacements of the master nodes retained in the reduction.

- $\mathbf{F}_{\mathbf{e x}}$ is the vector of external excitation acting on the blade airfoil, see Fig. 1(b).

- $\mathbf{F}_{\mathbf{c}}$, is the vector of centrifugal forces pushing the strip against the blade platform, see Fig. 1(b).

- $\mathbf{F}_{\mathbf{n l}}$ is the vector of friction nonlinear forces generated between the coupled nodes strip-blade by their relative displacements. Eq. 1 is nonlinear due to the presence of $\mathbf{F}_{\mathbf{n l}}$ which depends on the relative displacement and velocity between strip and blades.

Equation (1) is written in the time domain and it can be converted in the frequency domain and solved by the well-known Multi-Harmonic Balance Method (MHBM) $[19,20,38,39]$. In this way the system of nonlinear second order differentiaequations (1) is turned into a set of nonlinear algebraic complex equations, obtaining sensible reductions in the computational times. This is possible because the external excitation $\mathbf{F}_{\mathbf{e x}}$ is periodic (sinusoidal). Consequently, also the displacements $\mathbf{x}$ and the contact forces $\mathbf{F}_{\mathbf{n l}}$ are assumed to be periodic and are approximated by Fourier series. The presence of frictioninduced nonlinearities will produce displacements and contact forces which are still periodic but not perfectly sinusoidal (hence the need for the MHBM, rather than the simple HBM, to ensure an adequate harmonic support).

\section{Contact Model}

The contact model allows cthe nonlinear forces, $\mathbf{F}_{\mathbf{n l}}=$ $\left[T_{x}, N, T_{y}\right]$, generated by the relative displacements between strip and blade nodes. A 3-D contact model [40] is normally adopted in literature. In the 3-D model, the contact

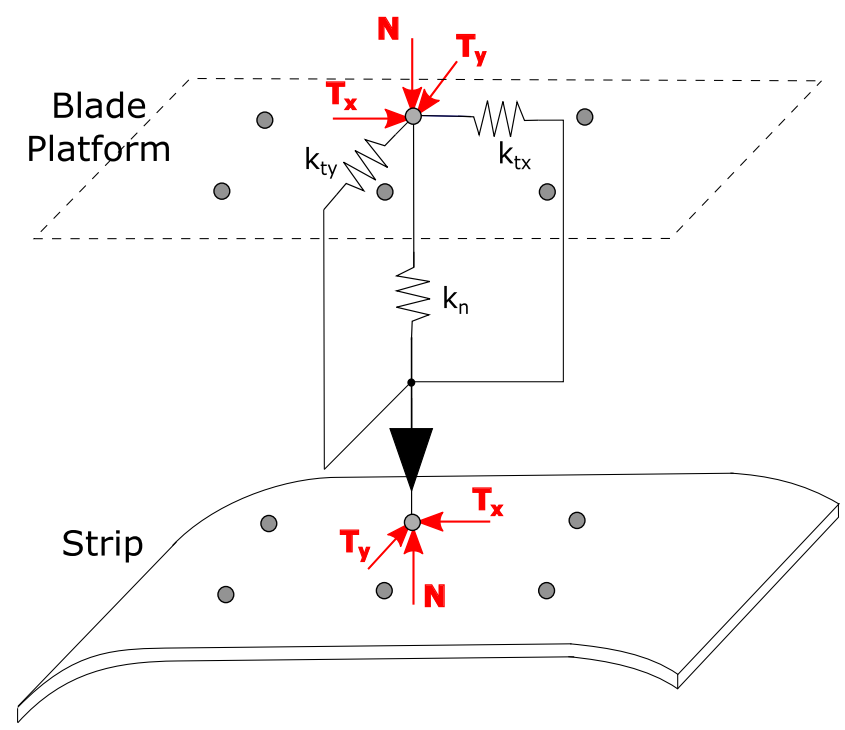

Fig. 2 The contact model 
Fig. 3 Scheme of the solution procedure

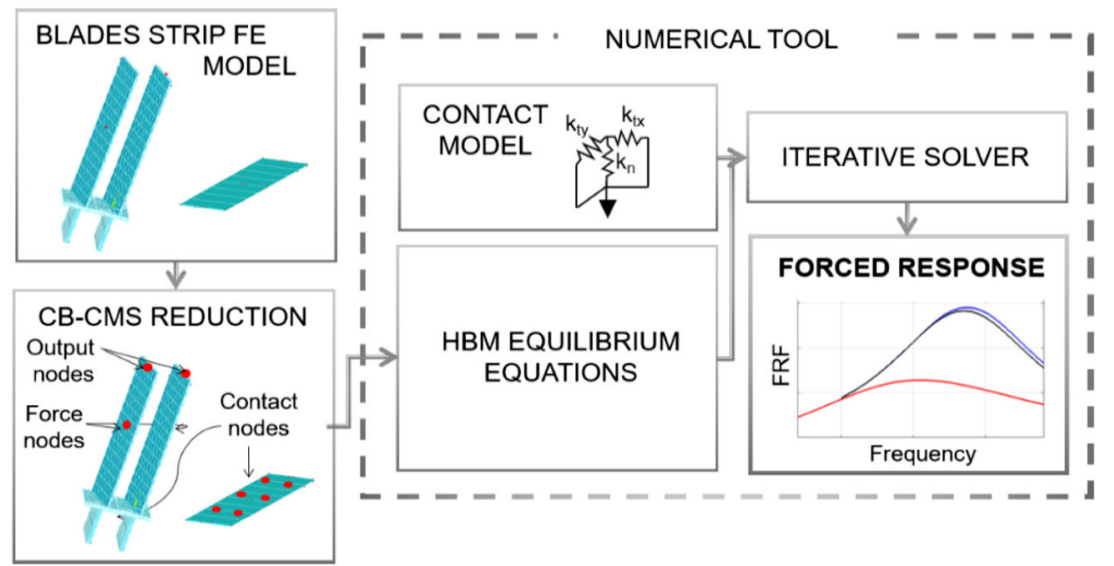

elasticity is approximated with three springs acting between each node of the strip and of the blade as shown in Fig. 2.

The contact stiffness values assigned to each contact node (i.e. local contact stiffness, $k_{n}, k_{t x}$ and $k_{t y}$ ) depend on the contact stiffness of the interface and on the number of contact nodes chosen to represent it. For instance, for $k_{n}: k_{n}=k_{n g} / N_{c}$, where $k_{n}$ and $k_{n g}$ are the local and global normal contact stiffness respectively, and $N_{c}$ is the total number of the couples of nodes which approximate the contact between strip and blade platforms. The same relationship holds for $k_{t x}$ and $k_{t y}$. If the contact state is "stick", the relation between forces and displacements at the contact is linear and governed by the contact springs described above. If the contact state is "slip", normal and tangential forces are linked by a proportionality constant which can be positive or negative depending on the direction of slipping (i.e. Coulomb's law of friction applies, $T=$ $\pm \mu N$ ). If the contact state is "lift-off", contact forces are zero as the contact nodes are separated.

\section{Numerical Calculation Procedure}

The numerical calculation is performed through a stateof-the-art numerical code based on the Multi-Harmonic Balance Method (MHBM) for an efficient solution of the equilibrium equations. A full description of this numerical tool is found in [22]. The code, originally developed for solid dampers which are considered as rigid bodies, was here adapted for strip dampers modeled using FE. A representative scheme of the code is shown in Fig. 3. A few notable features of the code are:

- implementation of the CB-CMS reduction technique to reduce the size of the FE model without loss of accuracy or relevant information [21];

- implementation of a state-of-the-art contact model, described in the previous section, to take into account the presence of friction. The contact model is implemented in the time domain to ensure an adequate representation of all contact situations (including slip and lift-off) and the AFT (Alternative Frequency Time) method [43] is applied to switch between frequency and time domain. Contact parameters (i.e. contact stiffnesses and friction coefficient) are needed as input by the code [41, 42];

- presence of the analytical Jacobian computation in the iterative solver to ensure efficiency [20, 22, 44];

- presence of the in-house Jacobian Alert Algorithm ${ }^{2}$ to ensure an accurate solution obtained in a timely manner [22].

The main output of the numerical tool is the forced response of the structure under different excitation levels, a vital indication in the blade-strip design process.

A calculation example of the forced response for the simplified system of Fig. 1(b) (i.e. two dummy blades coupled by a strip damper) is shown in Fig 4. The contact interfaces are assigned contact stiffness values $k_{n g}=1000$ $\mathrm{N} / \mu \mathrm{m}$ and $k_{t g}=233 \mathrm{~N} / \mu \mathrm{m}$ and a friction coefficient $\mu=0.6$. These values have been chosen, for lack of a better alternative, based on the authors' previous experience on rigid dampers [41] subjected to contact pressures similar to the strip's [45].

The present study is focused on the second mode of the system, i.e. the blade neck-bending mode shape. The choice is motivated by the fact that this mode is largely influenced by the presence of the strip. In fact, the strip acts as a constraint under the blade platform thus affecting considerably the motion of the neck. On the contrary the

\footnotetext{
${ }^{2}$ The Jacobian Alert Algorithm allows the user to run the MHBM simulation with a small harmonic support and gives a warning (hence the term "Alert") only if the error grows above a user-defined threshold, thus prompting the user to increase it to ensure accurate results. It is a cheap and effective alternative to standard convergence studies, and essential whenever severe nonlinearities are present (e.g. slipping or lift-off of contact points).
} 
first mode shape is pure airfoil-bending and therefore the strip does not alter significantly the system behavior. This difference between the two modes is easily quantified by comparing the relative motion of the platform kinematics with that of the airfoil. The first pure airfoil-bending mode sees a relative motion between neighboring platforms which is only $0.003 \%$ of the reference airfoil motion, while the second neck-bending mode sees a relative platform motion which is $0.13 \%$ of the reference airfoil motion.

In Fig. 4, Frequency Response Functions (FRFs), calculated at different excitation forces, show the typical trend in presence of friction damping. As the amplitude of the excitation force increases from $F_{e x}=0.1 \mathrm{~N}$, the contact may start to slip. As a result, the FRF amplitude decreases since more damping is introduced in the system and the natural frequency moves toward left since with slipping at the contact the system becomes less stiff. For very high values of the excitation force $(300 \mathrm{~N})$ the FRF is almost overlapped to the free FRF curve (linear case obtained for the blades without strip). In this case the value of the contact forces is zero and this means that almost all contact points are in lift off: the excitation force is so high that the strip loses contact with the blade. In fact, the present test case sees the two blades vibrating "In-Phase": the resulting platform kinematics, as shown in [45], causes the strip to detach and consequently its effect on the blades is minimized.

As stated in the introduction, no information on the contact parameters of flexible dampers is available. For this reason, the authors explored the influence of the variation of contact parameters on the FRF. Figure 5(a) shows the FRFs calculated in the case of strip stuck between the two blades, but with different values of the contact stiffness $k_{n}$ and $k_{t}$. The green curve in the middle is the same green curve of Fig. 4 and it is calculated with nominal values of $k_{n}$ and $k_{t}$. The other green curves are calculated by assuming respectively $10 k_{n}, 10 k_{t}$ and $0.1 k_{n}, 0.1 k_{t}$. This admittedly large range of values has been chosen by the authors to take into account the sample-to-sample variability observed on solid dampers (i.e. almost one order of magnitude [41]) and the uncertainty caused by the lack of experimental evidence on strip dampers. It is observed that the contact stiffness values have a strong influence on the FRF, in particular on the resonance frequency value. In the present example the strip introduces a $7 \mathrm{~Hz}$ frequency shift (with respect to the free blades) using the nominal values of $k_{t}$ and $k_{n}$ : the frequency shift can become twice as much if the contact springs are multiplied by 10 , or become negligible if $0.1 k_{n}$, $0.1 k_{t}$ are used.

Figure 5(b) explores the influence of the friction coefficient, $\mu$, on the FRFs for a case where the contacts slip during the period of vibration. Once again, it is observed that the predicted value of the FRF amplitude is different for the different $\mu$ values. For a given value of excitation force $\left(F_{e x}=20 \mathrm{~N}\right.$ in Fig 5(b)), assuming $\mu=0.6$ instead of $\mu=0.1$ can change the calculated amplitude of $50 \%$.

\section{The Need for a Direct Experimental Investigation}

In the previous section, it was shown that the calculation of blades with strips can be performed using the wellknown existing numerical techniques tested on blades with
Fig. 4 Nonlinear forced response of a system with two dummy blades and a strip damper, for different excitation force amplitudes $|F e x|$ and friction coefficient $\mu=0.6$

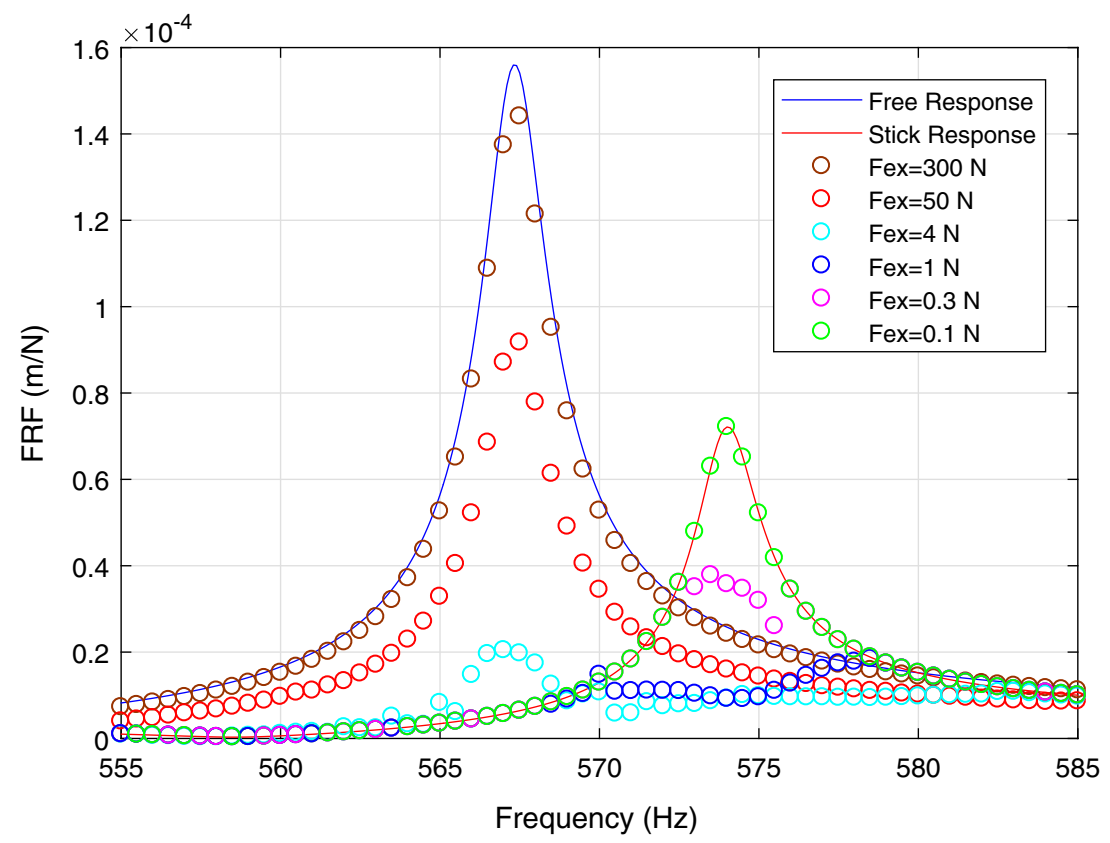



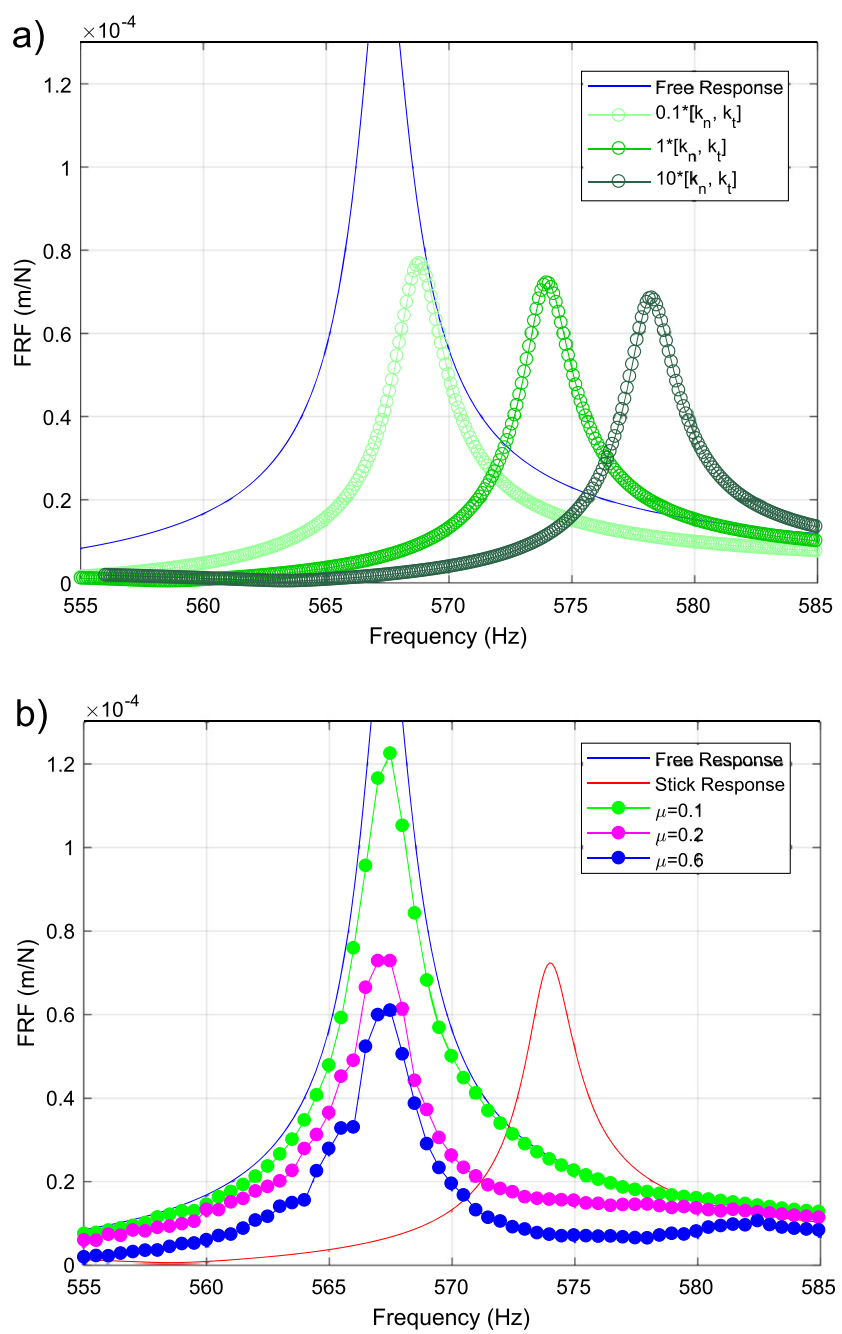

Fig. 5 Numerical forced response of a system with two dummy blades and a strip damper: (a) Influence of contact stiffness for $|F e x|=0.1 \mathrm{~N}$; (b) Influence of friction coefficient for $|F e x|=20 \mathrm{~N}$

solid dampers and shrouds. It was also shown that contact parameters values can change significantly the numerical prediction of the FRFs (see Fig. 5). The most challenging and pressing target, according to these authors, is the determination of the contact parameters $\left(k_{n}, k_{t}\right.$ and $\mu$ ) values to be given as input to the numerical tool.

The problem of the determination of realistic values of the contact parameters is well known in literature $[12,36]$ and it is an issue that it is not fully solved yet. Furthermore, to the authors' knowledge, there are no existing investigations on the estimation of the stripblade contact parameters, and no direct assessments of the adequacy of the contact model in this novel context.

In detail, the peculiarities of the strip (flexible) damper contact-case are the following:

- Modeling. The strip damper has a very low mass: as a consequence, the contact pressure induced by the centrifugal force is quite low (of the order of 0.3 $\mathrm{MPa}$ ). Results available in literature obtained for solid dampers at realistic centrifugal forces are obtained at much higher contact pressures (10-30 MPa is a typical range). As there is an evidence that contact parameters of conforming contacts are heavily influenced by contact pressure [14], values obtained for solid dampers may not be used for the flexible damper case.

- Testing. The strip damper is very flexible: the test rigs found in literature for the determination of contact parameters [12, 24, 42] apply contact pressure on one of the contacting bodies by means of a set of wires and pulleys connected to dead weights. The strip damper cannot be loaded by wires due to its high flexibility. The wires would deform the strip thus producing unrealistic contact conditions. The appropriate loading system for a strip damper should simulate the uniform pressure distribution provided by the centrifugal force, possibly without any contact with the strip.

By using their experience about direct experimental investigation of friction parameters on solid dampers, the authors propose in this paper the design of a new test rig, the first one specifically tailored for strip dampers. The test rig should allow the measurement of both the FRFs of a blade in contact with the strip and, at the same time, the hysteresis cycles at the strip-blade contact. Contact parameters (i.e. contact stiffness and friction coefficient) can be extracted from force signals and hysteresis cycles through an in-house data processing technique already applied with success on solid dampers data $[13,33]$. The test rig here presented has not yet been assembled and tested in total, but its key components have been designed, machined and tested separately.

\section{Definition of the Test Rig Requirements}

As pointed out in the previous section the peculiarity of the strip damper is to be flexible and very light. The following technical specifications are then required of the test rig:

- the test rig should be non-rotating in order to allow the accurate measurement of forces and displacement at the contact. This implies that the centrifugal force, generated only in rotating conditions, must be simulated in a different manner;

- the loading system simulating the centrifugal force on the strip should not be in contact with the strip in order to avoid influence on the strip stiffness and overall system dynamics;

- the hysteresis cycles at the blade-strip contact should be measured directly in order to assess the adequacy of 
the contact model and ultimately derive its calibration parameters;

- contact pads on the blade platform should be replaceable in order to be manufactured with different materials;

- the blade should be excited with a step-sine excitation force with constant amplitude. Since the system is nonlinear, a precise knowledge of the amplitude of the force exciting the system is required.

The test rig is designed to be assembled in two different configurations as sketched in Fig. 6.

The two black boxes $\mathrm{M}$ and $\mathrm{L}$ in Fig. 6 are key components that must be purposely designed. Box $\mathrm{M}$ is the measurement system of the hysteresis cycles at the blade-strip contact. This means that box $\mathrm{M}$ should include a system to measure the contact forces and a system to measure the tangential relative displacement between strip and blade platform.

Box $\mathrm{L}$ is the load system simulating the centrifugal force on the strip, this system should apply a pressure on the strip without direct contact.

The two different configurations in Fig. 6 have been designed for the collection of data in two different conditions, in detail:

a In configuration a) one blade coupled with one strip is tested and the hysteresis cycle can be directly measured on the right side where the strip is in contact with the box $\mathrm{M}$.

b In configuration b) two blades with one strip between them are tested, in this case the measurement of the hysteresis cycles on the strip is no more possible, but the FRFs of the two blades in contact with the strip can be measured.

Configuration a) is the fundamental one since it allows the measurement of the contact parameters to be given as input to the numerical code. Configuration b) allows a couple of blades to be tested in the in-phase (IP) and outof-phase (OOP) bending modes of vibration, considered as a benchmark to test the results of the numerical code. In detail, with reference to the case analyzed in the section "Numerical calculation procedure", the neckbending modes will be of particular interest, since the strip influence will be at its maximum. The FRFs measured in configuration b) for different excitation and centrifugal forces, can be compared to the numerical FRFs (like those of Fig. 4) calculated using the contact parameters obtained by the hysteresis cycles measured in configuration a).

\section{The Test Rig Mechanical Structure}

The basic structure of the test rig (Fig. 7(a)) consists of a clamping hydraulic machine, which constrains the blades, and a rigid structure, which holds the measurement devices and the excitation shaker.

The clamping system is shown in Fig. 7(a). A hydraulic piston moves a clamping head that constrain the item to be tested. In the present study a dummy blade is constrained, as shown in Fig. 7(b).

The dummy blade (Fig. 7(b)) is in one-piece with a prismatic basis where the clamping force is applied. The blade platform is designed with a replaceable contact pad (in contact with the strip) to test different materials.

The strip damper has been obtained using a guillotine cutter on a ground stainless steel cold-rolled sheet. This choice avoids bending of the strip and ensures clean edges (then further fine ground). Its main dimensions are shown in Fig. 8. They have been chosen, the $0.4 \mathrm{~mm}$ thickness in particular, to mimic those of a real sealing strip mounted on a turbine for power generation known to the authors. The same test case has been used to estimate the necessary contact pressure. The distance of the strip from the disk
Fig. 6 Test rig different configurations: (a) configuration for the hysteresis cycles measurements; (b) configuration for the FRF measurements
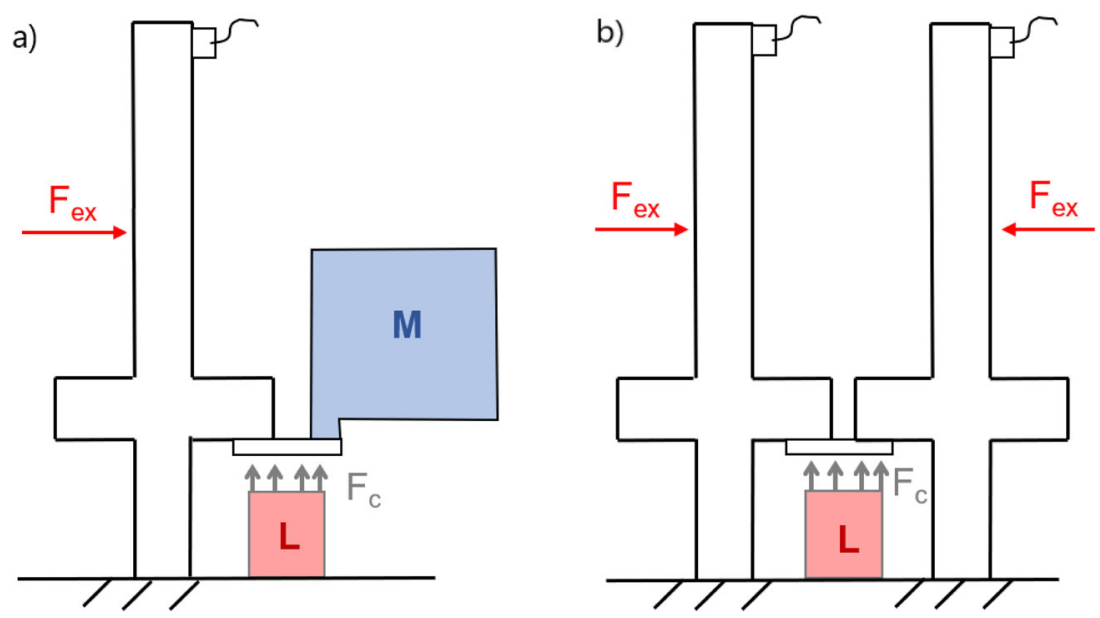
Fig. 7 (a) Test rig basic structure; (b) dummy blade clamped in the test rig
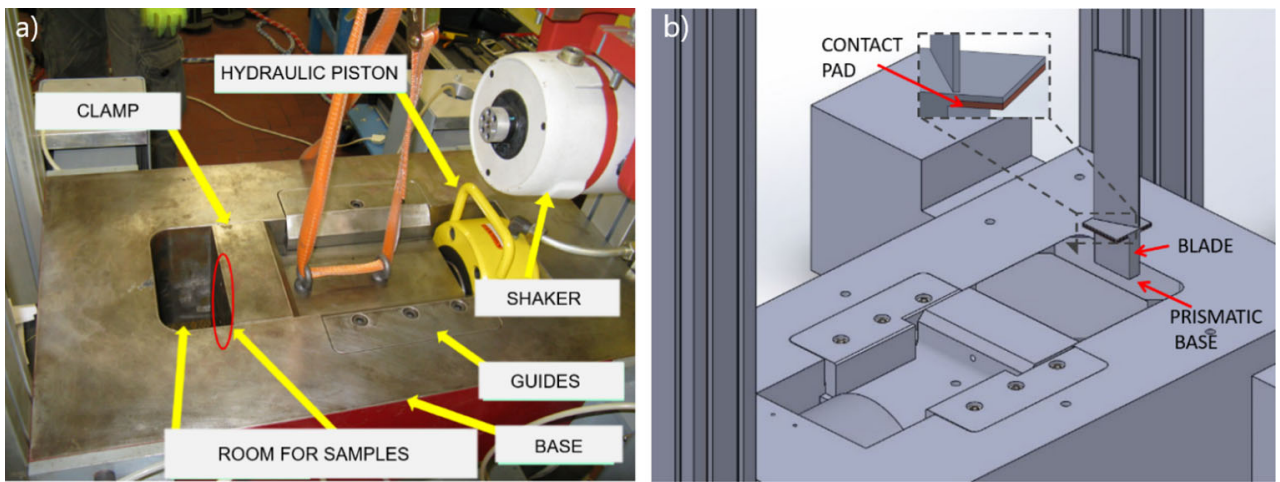

axis is approximately $1 \mathrm{~m}$, which yields a centrifugal load of $\approx 180 \mathrm{~N}$ and, consequently, a contact pressure of $\approx 0.36$ $\mathrm{MPa}$.

\section{The Contact Forces Measuring System}

The system to measure the contact forces has been previously designed, set up and tested on a test rig for the direct experimental investigation of solid UPDs and their contact with the corresponding platforms [33, 34].

The force measurement is based on an L-shaped structure. The $\mathrm{L}$ structure is connected to two piezo force transducers, here termed $t_{1}$ and $t_{2}$, sketched in Fig. 9(a). The $\mathrm{L}$ structure is designed so that the component of the contact force $F_{1}$ is almost totally measured by the transducer $t_{1}$ and the force component $F_{2}$ by the transducer $t_{2}$. This decoupling of the two transducers is obtained by designing the $\mathrm{L}$ structure with a stiffness in the longitudinal direction
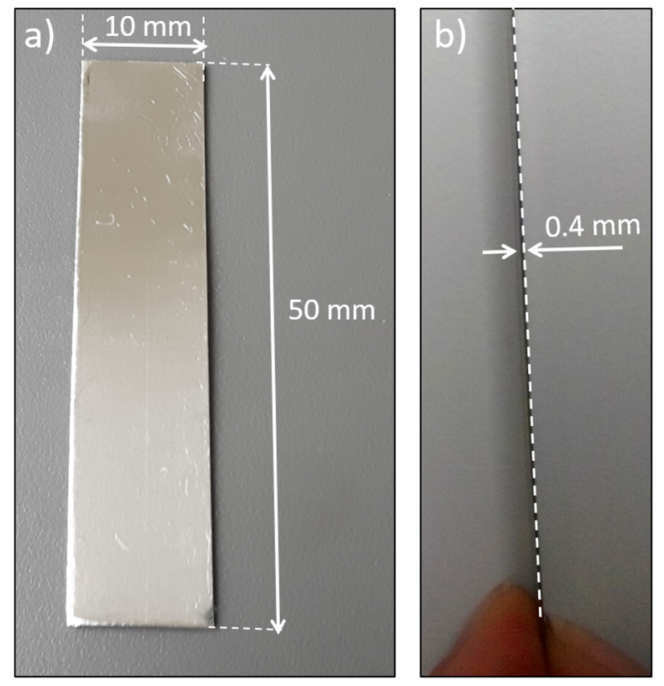

Fig. 8 (a) Strip damper top view and main dimensions. (b) Strip damper side view and thickness. A dashed line shows its planarity of each limb $\left(K_{11} \sim K_{22}\right)$ much higher than the transversal one $\left(K_{12} \sim K_{21}\right)$, i.e. $\left(K_{11} \sim K_{22}\right)>>\left(K_{12} \sim K_{21}\right)$.

To practically obtain this high longitudinal-to-transver-se stiffness ratio, each leg of the L shaped is composed of two thin parallel strips as shown in Fig. 9(b). The two limbs are perpendicular to each other and their axes intersect in a point as close as possible to the contact surface. In [34] it was demonstrated that the force on transducer, $R_{n 1}=0.995 F_{1}$ and $R_{n 2}=0.995 F_{2}$. This accuracy can decrease to 0.991 if the intersection of the axes of the two limbs is at $\pm 1.5 \mathrm{~mm}$ from the center of the contact area.

The system with two limbs is applied to the strip test rig in configuration a), as sketched in Fig. 6(a). The system is oriented so that the normal $(\mathrm{N})$ and tangential (T) components of the contact forces are separately detected by the two force transducers $t_{1}$ and $t_{2}$.

\section{The Test Rig Assembly}

A basic plate and a rigid support are added to the test rig configuration a), in order to ensure that the force measuring system is rigidly constrained to the structure. The final assembly of the test rig in the two configurations a) and b) is shown in Fig. 10.

\section{The Measurement of the Relative Displacement}

A differential vibrometer is arranged to measure the displacements between point A and point B in Fig. 11. Point $\mathrm{A}$ is on the insert connected to the $\mathrm{L}$ force measurement system, and point B is on the strip. The vibrometer used in this work can detect directly both velocities and displacements. Since, in this case, the quantities of interest are displacements, this function is mainly used. The scaling factor can be adjusted to meet the needs of measurement and is directly linked to the resolution. The measurements carried out in the previous tests with solid dampers used a 
Fig. 9 System for the measurement of contact forces:

(a) L-structure spring scheme;

(b) Measurement system applied

to the contact with the strip
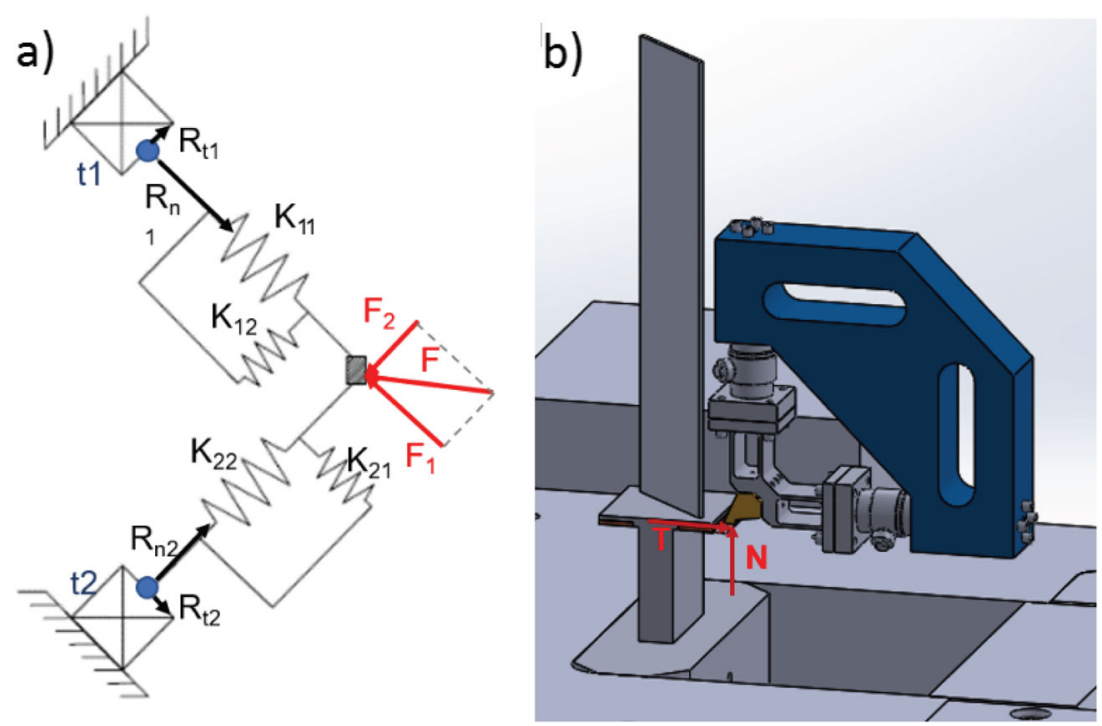

$20 \mu \mathrm{m} / \mathrm{V}$ scaling factor and a $0.08 \mu \mathrm{m}$ resolution. The order of magnitude of the relative displacement expected here (based on the numerical investigation described above) is $5 \mu \mathrm{m}$. The error on the relative displacement measurement estimated on the previous test rig is $0.08 \mu \mathrm{m}$.

\section{The Strip Loading System}

As stated above, the key component of this new test rig is the strip loading system used to simulate the centrifugal load on the strip. The strip loading system should then have the following, rather challenging, technical requirements:

- to be non-contact since the strip is very flexible. Any contact device can deform the strip and modify the blade-strip contact surface;

- to be capable of producing a uniform strip-blade pressure of the order of magnitude of the pressure induced by the centrifugal force in service. A typical realistic pressure value on the contact surface stripblade is $0.3 \mathrm{MPa}$;
- to be capable of guaranteeing a constant pressure for the time needed to measure contact hysteresis cycles and blade forced responses (about ten minutes).

The loading system proposed here is, to the authors' knowledge, the first one of its kind in this field. It uses compressed air. A first prototype has been produced and tested: relevant pictures are shown in Fig. 12. At the inlet in the red central box of Fig. 12(a), a pressure regulator controls the pressure and a manometer measures its value. The rectangular strip lies in the strip seat. The compressed air goes out through the output holes and lifts the strip in contact with a support, here simulating the blade platforms. A gap between the compressed air box and the strip, when the strip is in contact with the support (i.e. platforms), is necessary. This gap, set in this paper at $0.1 \mathrm{~mm}$, guarantees that, once the system is turned on, the strip will touch only the platforms and not any portion of the compressed air box. This gap also contributes to the loss of pressure (as will be shown below) and should therefore be kept as small as possible. It is then necessary to measure the effective contact pressure obtained at the strip-support interface for a given
Fig. 10 Final assembly of the test rig: (a) configuration a; (b) configuration $b$ a)

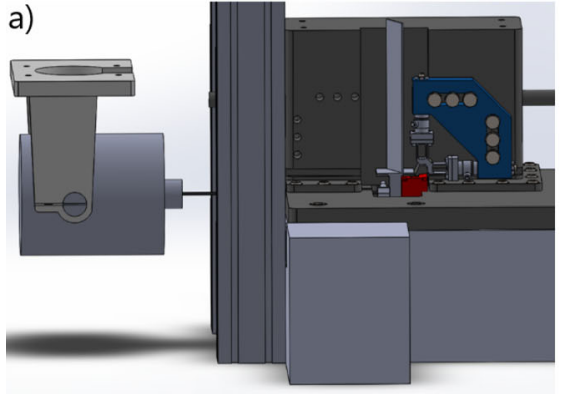

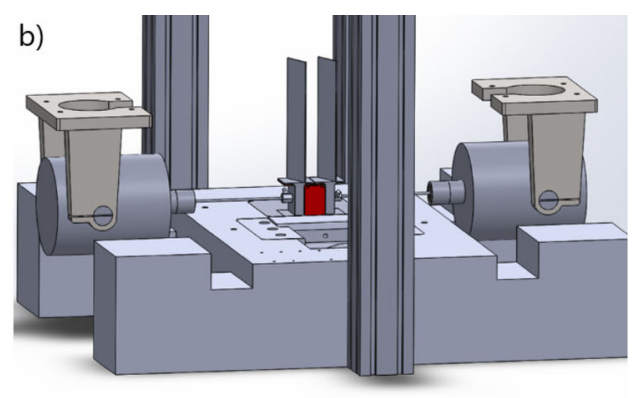


Fig. 11 Measurement system for the relative displacement strip damper-blade
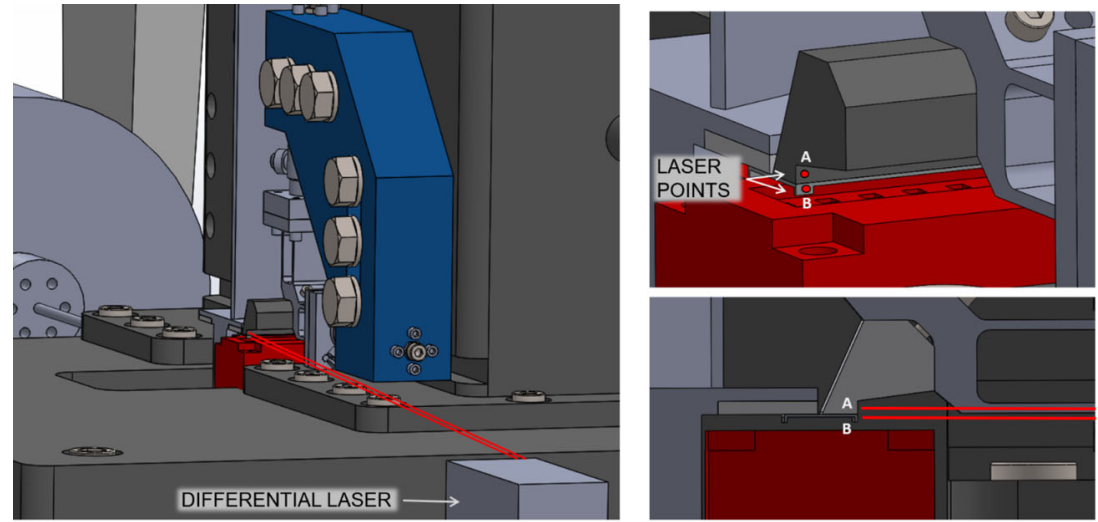

input of the inlet pressure. The investigation started with a $0.75 \mathrm{MPa}$ pressure at the inlet, the maximum value presently available in the laboratory.

The contact pressure is here measured by a LLLW Prescale Fujifilm film positioned between the strip and the support (see Fig. 12(b)). The measurement result is shown in Fig. 13(a). The value of the pressure on the contact area is obtained using the calibration scale provided by Fujifim. The color distribution is fairly uniform with darker spots, mainly present in the lower left corner. This darker region may be caused by local elastic deformations of the strip subjected to stronger air jets (the lower portion of the strip is also closer to the pressure inlet). The average pressure value is $0.28 \mathrm{MPa}$ with minor deviations across the contact patch $(\approx 77 \%$ of the area has a local pressure value in the [0.25$0.31] \mathrm{MPa}$ range). Since this contact pressure is slightly lower than the target $(0.36 \mathrm{MPa})$ the authors deemed useless performing tests at lower inlet pressure values. Achieving higher contact pressure values is possible by installing an inlet pressure increaser. Thanks to the pressure regulator this pressure value at the inlet can be kept constant for more than ten minutes, the time required for the measurement of the contact hysteresis cycles and of the blades forces response.

For the sake of comparison, the pressure distribution induced on the strip by the compressed air loading systems has been evaluated against that induced by a "standard" loading technique. The strip was then loaded using a rigid punch (which could be easily connected to dead weights). In this case the loading punch, with a cross section of the same shape and size $(10 \mathrm{~mm} \times 50 \mathrm{~mm})$ as the strip's, has been loaded using dead weights for a total of $20 \mathrm{~kg}$, to reproduce the expected uniform pressure of $0.36 \mathrm{MPa}$. The punch pushes the strip against a perfectly flat granite table. Once again, the Prescale Fujifilm is used to assess the pressure distribution. Relevant results are shown in Fig. 13(b).

This system proved to be ineffective for two main reasons:

- it is in contact with the strip modifying the strip stiffness and the contact area;

- it does not apply a uniform pressure since, as shown in Fig. 13(b), the pressure is applied mainly on the strip edges with pressure peaks $>0.7 \mathrm{MPa}$.

Despite the planarity of the strip (see also Fig. 8(b)), the imperfect mating between strip and loading punch does not ensure a uniform contact pressure. This may be due to the loading punch configuration or to oscillations introduced by the experimenter when loading the deadweights. This comparison further convinced the authors of this paper that classical dead weight-based systems should be discarded in the case of strip dampers, while a compressed airbased loading system is a viable solution for the following motives:
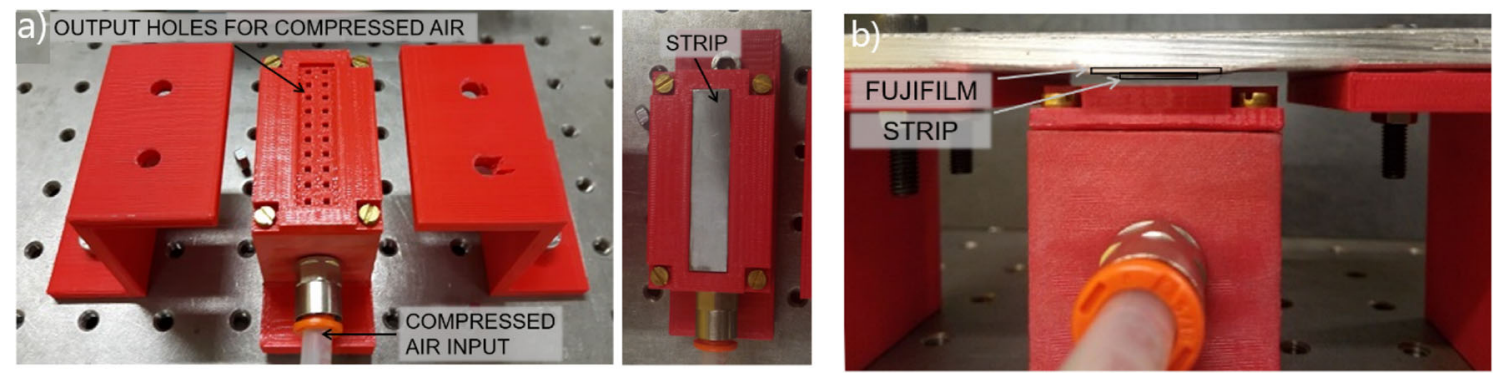

Fig. 12 (a) Strip loading system by compressed air; (b) Fujifilm between strip and support loaded by compressed air 
Fig. 13 (a) Pressure measurement by Prescale Fujifilm in the case of compressed air; (b) pressure measurement by Prescale Fujifilm in the case of dead weights applied on the strip
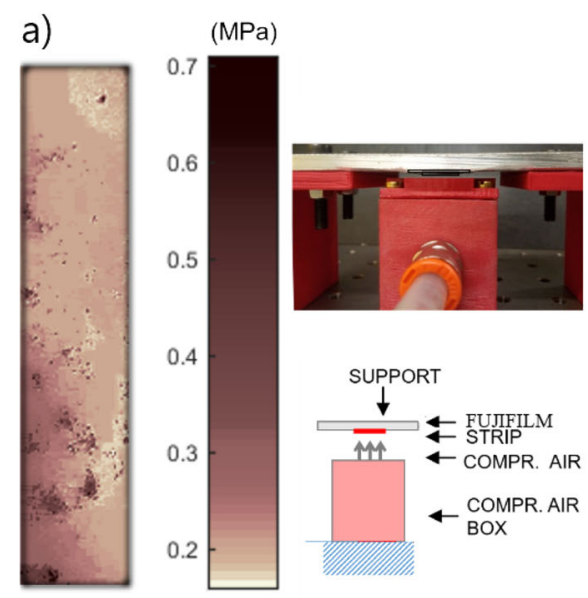
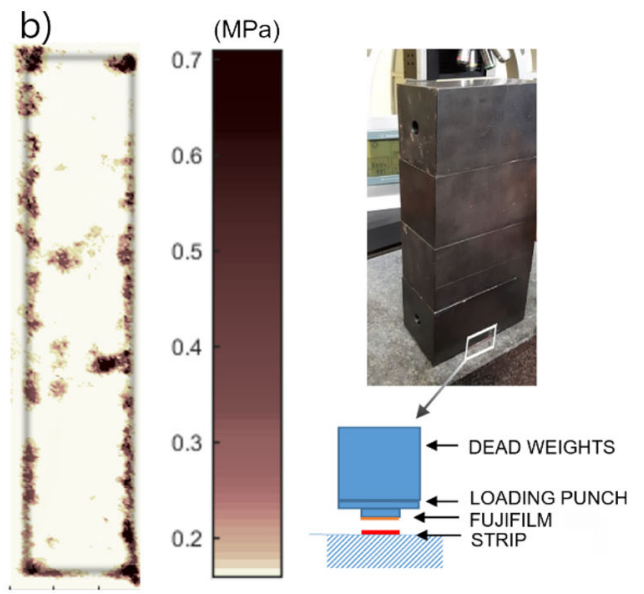

- does not touch the strip, and therefore does not influence the strip stiffness and contact area;

- is able to provide a uniform and continuous pressure at the blade-strip contact, with a realistic pressure value (about $0.3 \mathrm{MPa}$ ).

\section{Determination of Contact Parameters and Test of the Force and Relative Displacement Measuring Systems}

The L force measuring system (Fig. 9) and relative displacement measurement system (Fig. 11) have both been tested on a rig for the direct experimental investigation for solid dampers [34]. Figure 14 shows a sample of results obtained at $5 \mathrm{~Hz}$ (frequencies up to $600 \mathrm{~Hz}$ have been successfully tested as well) and at a contact pressure of 2 MPa. Fig. 14 also shows an example of the data processing technique used to determine contact parameters (using data coming from solid dampers [41]).

In detail,

- friction coefficients will be obtained through the analysis of the Tangential/Normal force ratio (see Fig. 14(a)). When the $\mathrm{T} / \mathrm{N}$ signal is constant in time and equal to a maximum the contact is in slip and $\mu= \pm T / N$ where $\mu$ is the friction coefficient according to Coulomb's definition. If $\mathrm{T} / \mathrm{N}$ is instead varying in time then the contact is in stick.

- Tangential contact stiffness values will be estimated using the hysteresis cycle (Fig. 14(b)) which relates the relative tangential displacement at the contact to the corresponding component of the contact force $\mathrm{T}$. The slope of the hysteresis (obtained during a portion of the period when the $\mathrm{T} / \mathrm{N}$ ratio is varying, i.e. stuck contact) represents the tangential contact stiffness $k_{t}$. a)

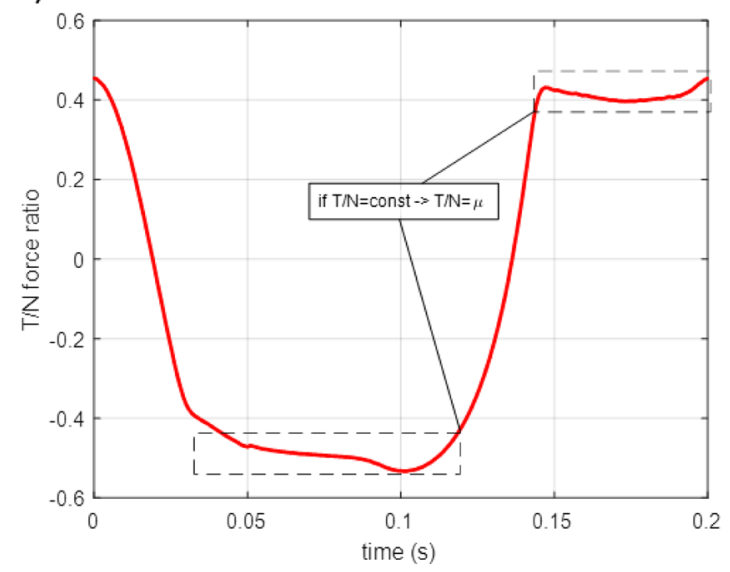

b)

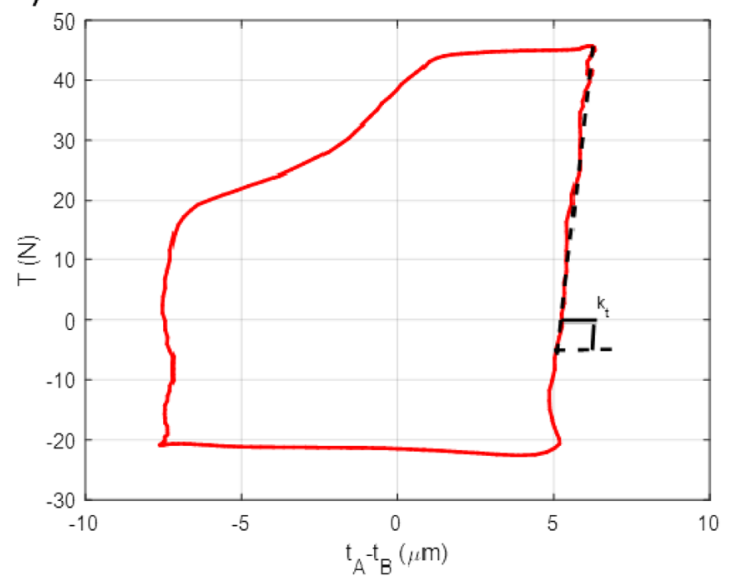

Fig. 14 Example of contact parameter determination starting from experimental evidence obtained from existing test rig for solid dampers: (a) Determination of friction coefficient starting from force measurement; (b) Determination of contact stiffness starting from the hysteresis cycle 
This method has been successfully applied to solid dampers [34] and the accuracy levels are more than adequate. In detail, force measurements are highly repeatable and with an accuracy of $1 \%$. This yields $\mathrm{T} / \mathrm{N}$ force ratios $(\mu)$ estimated with an error lower than $1 \%$. Similarly, contact stiffness values estimated on the same contact are very repeatable and estimated with an error $<5 \%$. The authors are aware that force ranges typical of strip dampers may be different from those already experienced with solid dampers. This does not impair the accuracy of the method as the chosen load cells (Kistler 9323AA), which ensure an error lower than $1 \%$ of the full scale range, can be set according to different full scale ranges. In detail, the $200 \mathrm{~N}$ full scale range is perfectly adequate to test the strip used in this paper (centrifugal force at approximately $180 \mathrm{~N}$ as discussed above).

\section{Conclusions}

The paper goal is to offer a solid contribution in the field of flexible damper testing. This subject is still largely unexplored, but nevertheless essential to trustworthy predictions of the nonlinear forced response of turbine blades.

It is here shown that the computed frequency response of two blades with a strip damper strongly depends on the chosen contact parameters values (friction coefficient, normal and tangential contact stiffnesses) at the stripblade contact. Testing flexible dampers in a controlled environment (e.g. non-rotating rig) to the purpose of estimating contact parameters is challenging, as it requires providing a uniform centrifugal load on the strip without modifying its stiffness and contact conditions.

The design of a novel test rig tailored on strip dampers is here proposed. The test rig design includes a measurement system for contact forces ( $1 \%$ accuracy) and for relative displacements at the contacts (0.08 $\mu \mathrm{m}$ accuracy) which was already tested on a previous rig for solid UPDs.

If the measurement system of contact forces and displacements is borrowed by a previous test rig, the real novel key element of this test rig is the strip loading system which simulates the centrifugal force. The loading system here proposed is based on compressed air. The first prototype has been built and tested. It proved to be suited to the purpose as:

- it produces an average pressure across the contact surface up to $0.28 \mathrm{MPa}$, which is a realistic value of the pressure on the strip in service during rotation;

- the pressure distribution is uniform, in fact $77 \%$ of the contact area shares a local pressure in the $\pm 10 \%$ range about the average value;
- it provides a pressure which is constant in time thanks to a pressure regulator of the compressed air at the inlet; - unlike classical punch and dead weight systems, it does not modify the contact conditions nor the strip stiffness.

For these reasons, the authors are convinced that the new loading system is a solid and viable solution in the frame of flexible damper testing and, above all, a necessary step in the path toward the creation of a trustworthy and predictive design tool.

Open Access This article is distributed under the terms of the Creative Commons Attribution 4.0 International License (http:// creativecommons.org/licenses/by/4.0/), which permits unrestricted use, distribution, and reproduction in any medium, provided you give appropriate credit to the original author(s) and the source, provide a link to the Creative Commons license, and indicate if changes were made.

\section{References}

1. Szwedowicz J (2008) Bladed disks: nonlinear dynamics. Structural design of aircraft engines: key objectives and techniques Belgium, Von Karman Institute Lecture Series, Seinturier \& Paniagua editors

2. Srinivasan AV (1997) Flutter and resonant vibration characteristics of engine blades. J Eng Gas Turbines Power 119(4):742

3. Petrov EP (2008) Explicit finite element models of friction dampers in forced response analysis of bladed disks. J Eng Gas Turbines Power 130(2):022502

4. Afzal M, Lopez AI, Kari L (2016) Investigation of damping potential of strip damper on a real turbine blade. In: Paper GT201657230 Proceedings of ASME Turbo Expo 2016: Turbomachinery Technical Conference and Exposition

5. Laxalde D, Thouverez F, Sinou JJ, Lombard JP (2007) Qualitative analysis of forced response of blisks with friction ring dampers. Eur J Mech A Solids 26(4):676-687

6. Seunghun B, Bogdan E (2017) Reduced-order modeling of bladed disks with friction ring dampers. J Vib Acoust 139(6):061011

7. Fantetti A (2017) Calculation of the forced response of a turbine bladed disk with strip dampers. Master's Thesis, University of Illinois at Chicago

8. Sever IA, Petrov EP, Ewins DJ (2008) Experimental and numerical investigation of rotating bladed disk forced response using underplatform friction dampers. J Eng Gas Turbines Power 130:42503

9. Bessone A, Toso F, Berruti TM (2015) Investigation on the Dynamic Response of Blades With Asymmetric Under Platform Dampers. In: Proceedings of ASME Turbo Expo 2015: Turbine Technical Conference and Exposition

10. Armand J, Pesaresi L, Salles L, Schwingshackl CW (2016) A multi-scale approach for nonlinear dynamic response predictions with fretting wear. J Eng Gas Turbines Power 139:2

11. Pesaresi L, Salles L, Jones A, Green JS, Schwingshackl CW (2017) Modelling the nonlinear behaviour of an underplatform damper test rig for turbine applications. Mech Syst SignalProcess 85:662-679

12. Gastaldi C, Gola MM (2015) A random sampling strategy for tuning contact parameters of underplatform dampers. In: Proceedings of ASME Turbo Expo 2015: Turbine Technical Conference and Exposition 
13. Gastaldi C, Gola MM (2016) On the relevance of a microslip contact model for under platform dampers. Int J Mech Sci 115116:145-156. https://doi.org/10.1016/j.ijmecsci.2016.06.015

14. Gastaldi C, Gola M (2016) Pre-optimization of asymmetrical underplatform dampers. J Eng Gas Turbines Power 139(GTP-161229):012504. https://doi.org/10.1115/1.4034191

15. Panning L, Sextro W, Popp K (2000) Optimization of interblade friction damper design. Manufacturing Materials and Metallurgy Ceramics Structures and Dynamics Controls, Diagnostics and Instrumentation Education 4 ASME https://doi.org/10.1115/200 0-GT-0541

16. Panning L, Sextro W, Popp K (2002) Optimization of the contact geometry between turbine blades and underplatform dampers with respect to friction damping. Turbo Expo 2002, 4:Parts A and B. ASME. https://doi.org/10.1115/GT2002-30429

17. Panning L, Popp K, Sextro W (2004) Asymmetrical underplatform dampers in gas turbine bladings: Theory and application ASME Turbo Expo 2004: Power for Land, Sea, and Air. https://doi.org/10. 1115/GT2004-53316

18. Sanliturk KY, Ewins DJ (1996) Modelling two-dimensional friction contact and its application using harmonic balance method. J Sound Vib 193(2):511-523

19. Cardona A, Coune T, Lerusse A (1994) A multi-harmonic method for nonlinear vibration analysis. Int $\mathrm{J}$ Numer Methods Eng 37:1593-1608

20. Petrov EP, Ewins DJ (2003) Analytical formulation of friction interface elements for analysis on nonlinear multi-harmonic vibrations of bladed disks. J Turbomach 125:364-371

21. Craig RR, Bampton MCC (1968) Coupling of substructures for dynamic analyses. AIAA J 6(7):1313-1319

22. Gastaldi C, Berruti TM (2017) A method to solve the efficiency accuracy trade-off of multi-harmonic balance calculation of structures with friction contacts. Int J Non-Linear Mech 92:25-40. https://doi.org/10.1016/j. ijnonlinmec.2017.03.010

23. Botto D, Campagna A, Lavella M, Gola MM (2010) Experimental and numerical investigation of fretting wear at high temperature for aeronautical alloys. ASME Turbo Expo: Parts A and B. 1353-1362

24. Schwingshackl CW, Petrov EP, Ewins DJ (2012) Measured and estimated friction interface parameters in a nonlinear dynamic analysis. Mech Syst Signal Process 28:574-84

25. Schwingshackl CW, Petrov EP, Ewins DJ (2012) Effects of contact interface parameters on vibration of turbine bladed disks with underplatform dampers. J Eng Gas Turbines Power 134(3):032507

26. Botto D, Lavella M (2015) A numerical method to solve the normal and tangential contact problem of elastic bodies. Wear 330-331:629-635

27. Armand J, Pesaresi L, Salles L, Schwingshackl CW (2016) A multiscale approach for nonlinear dynamic response predictions with fretting wear. J Eng Gas Turbines Power 139(2):022505022505-7. https://doi.org/10.1115/1.4034344

28. Lavella M, Botto D, Gola MM (2011) Test rig for wear and contact parameters extraction for flat-on-flat contact surfaces. ASME/STLE 2011 Joint Tribology Conference 307-309
29. Berruti T, Firrone CM, Gola MM (2011) A test rig for noncontact traveling wave excitation of a bladed disk with underplatform dampers. J Eng Gas Turbines Power 133:1-7

30. Botto D, Lavella M (2012) Measurement of contact parameters of flat on flat contact surfaces at high temperature. ASME Turbo Expo: Parts A and B. 1325-1332

31. Berruti T, Firrone CM, Pizzolante M, Gola M (2007) Fatigue damage prevention on turbine blades: study of underplatform damper shape. Key Eng Mater 347:159-164

32. Berruti T, Maschio V (2012) Experimental investigation on the forced response of a dummy counter-rotating turbine stage with friction damping. J Eng Gas Turbines Power 134(12):122502

33. Botto D, Gastaldi C, Gola MM, Umer M (2017) An experimental investigation of the dynamics of a blade with two Under-Platform dampers. J Eng Gas Turbines Power ISSN 0742-4795

34. Botto D, Umer M (2017) A novel test rig to investigate under-platform damper dynamics. Mech Syst SignalProcess 100:344-359

35. Thomas DL (1979) Dynamics of rotationally periodic structures. Int J Numeral Meth Eng 14(1):81-102

36. Zucca S, Firrone CM, Gola M (2012) Modeling underplatform dampers for turbine blades: a refined approach in the frequency domain. J Vib Control 19(7):1087-1102

37. Firrone CM, Zucca S (2011) Modeling priction contacts in structural dynamics and its application to turbine bladed disks. Numer Anal - Theory Appl 14:301-334

38. Sanliturk KY, Ewins DJ, Stanbridge AB (2001) Underplatform dampers for turbine blades: theoretical modeling, analysis and comparison with experimental data. J Eng Gas Turbines Power 123:919-929

39. Petrov EP, Ewins DJ (2007) Advanced modeling of underplatform friction dampers for analysis of bladed disk vibration. J Turbomach 129:143-150

40. Yang BD, Menq CH (1998) Characterization of 3D contact kinematics and prediction of resonant response of structures having 3D frictional constraint. J Sound Vib 217:909-925

41. Gastaldi C (2017) Vibration control and mitigation in Turbomachinery. PhD Thesis, Politecnico di Torino

42. Lavella M (2016) Contact properties and wear behaviour of nickel based superalloy ren 80. Metals 6(7):159. https://doi.org/10.3390/ met6070159

43. Cameron T, Griffin J (1989) An alternating frequency/time domain method for calculating the steady-state response of nonlinear dynamic system. J Appl Mech 56(1):149-154. https://doi.org/10.1115/1.3176036

44. Siewert C, Panning L, Wallaschek J, Richter C (2010) Multiharmonic forced response analysis of a turbine blading coupled by nonlinear contact forces. J Eng Gas Turbines Power 132: 082501

45. Gastaldi C, Fantetti A, Berruti TM (2017) Forced response prediction of turbine blades with flexible dampers: the impact of engineering modelling choices. Appl Sci 8:34 